[3] Fleischman $R$ et al. Lancet 2017; 390: 457-6.

[4] Wollenhaupt et al. Arthritis Research \& Therapy (2019) 21:89

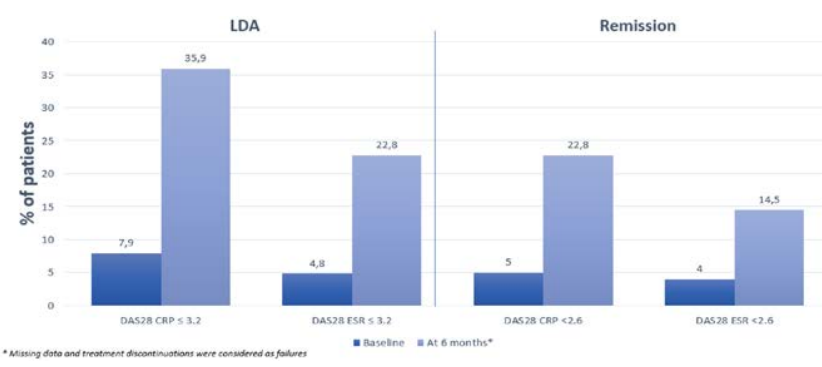

Figure 1. Patients with LDA or remission at baseline and 6 months

Table 1. Safety data

\begin{tabular}{lccc}
\hline Tolerability, $\mathbf{n}$ patients (\%) & $\mathbf{< 6 5}$ years, $\mathbf{n}=\mathbf{1 3 2}$ & $\mathbf{\geq 6 5}$ years, $\mathbf{n = 8 6}$ & Total $\mathbf{n}=\mathbf{2 1 8}$ \\
\hline$A E$ & $56(42.4)$ & $33(38.4)$ & $89(40.8)$ \\
Serious AE & $8(6.1)$ & $5(5.8)$ & $13(6.0)$ \\
Herpes Zoster & $4(3.0)$ & $2(2.3)$ & $6(2.8)$ \\
Infection & $27(20.5)$ & $15(17.4)$ & $42(19.3)$ \\
Severe infection & $2(1.5)$ & $2(2.3)$ & $4(1.8)$ \\
\hline
\end{tabular}

Acknowledgements: To all investigators involved in this study.

Disclosure of Interests: Cécile Gaujoux-Viala Speakers bureau: AbbVie; Amgen; Bristol-Myers Squibb; Celgene; Eli Lilly; Galapagos; Gilead Sciences, Inc.; Janssen; Medac; Merck-Serono; Mylan; Nordic Pharma; Novartis; Pfizer; Roche; Sandoz; Sanofi; and UCB, Consultant of: AbbVie; Amgen; Bristol-Myers Squibb; Celgene; Eli Lilly; Galapagos; Gilead Sciences, Inc.; Janssen; Medac; Merck-Serono; Mylan; Nordic Pharma; Novartis; Pfizer; Roche; Sandoz; Sanofi; and UCB, Andre BASCH Speakers bureau: Janssen, Novartis, Amgen, BMS, Abbvie, Lilly, Pfizer, MSD, UCB, Consultant of: Janssen, Novartis, Amgen, BMS, Abbvie, Lilly, Pfizer, MSD, UCB, Slim Lassoued: None declared, Fabienne COURY-LUCAS Consultant of: AbbVie, Bristol-Myers Squibb, Janssen, Lilly, MSD, Novartis and Pfizer, Grant/research support from: AbbVie, Biogen, Roche Chugai, Pfizer, and UCB, Meriem Kessouri Shareholder of: Pfizer, Employee of: Pfizer, Nadir Mammar Shareholder of: Pfizer, Employee of: Pfizer, Thierry Lequerre Consultant of: Abbvie, BMS, Boeringher, Lilly, Medac, MSD, Nordic Pharma, Novartis, Pfizer, Roche - Chugai, Sanofi, UCB, Carine Salliot Consultant of: Biogen, Lilly, Novartis, Roche Chugai, Pfizer

DOI: 10.1136/annrheumdis-2021-eular.870

\section{POS0660 CONCOMITANT USE OF STATINS IN FILGOTINIB- TREATED PATIENTS WITH RHEUMATOID ARTHRITIS}

P. C. Taylor ${ }^{1}$, C. Charles-Schoeman ${ }^{2}$, M. Alani ${ }^{3,4}$, M. Trivedi ${ }^{3}$, V. Castellano ${ }^{5}$ I. Tiamiyu ${ }^{3}$, D. Jiang ${ }^{6}$, L. Ye ${ }^{6}$, S. Strengholt ${ }^{7}$, M. Nurmohamed ${ }^{8}$, G. R. Burmester ${ }^{9}{ }^{1}$ University of Oxford, Botnar Research Center, Oxford, United Kingdom; ${ }^{2}$ UCLA Medical Center, Department of Medicine, Los Angeles, United States of America; ${ }^{3}$ Gilead Sciences, Inc., Clinical Research, Foster City, United States of America; ${ }^{4}$ University of Washington, Department of Rheumatology, Seattle, United States of America; ${ }^{5}$ Gilead Sciences, Inc. Rheumatology Therapeutic Area, Foster City, United States of America; ${ }^{6}$ Gilead Sciences, Inc., Biostatistics, Foster City, United States of America; ${ }^{7}$ Galapagos $B V$, Department of Rheumatology, Leiden, Netherlands; ${ }^{8}$ Amsterdam Rheumatology \& Immunology Center, Department of Rheumatology, Amsterdam, Netherlands; ${ }^{9}$ Charité - Universitätsmedizin Berlin, Department of Rheumatology and Clinical Immunology, Berlin, Germany

Background: The Janus kinase-1 preferential inhibitor filgotinib (FIL) improved rheumatoid arthritis (RA) signs and symptoms in phase $(\mathrm{P}) 3$ trials. ${ }^{1-3}$ RA elevates cardiovascular disease risk; statins are used to reduce risk.

Objectives: To assess safety of statin and filgotinib coadministration across the clinical program.

Methods: Patients (pts) meeting 2010 ACR/EULAR RA criteria in P2 DARWIN 1-2 (D1-2; NCT01888874, NCT01894516), P3 FINCH 1-3 (F1-3; NCT02889796, NCT02873936, NCT02886728), and long-term extensions DARWIN 3 and FINCH 4 (D3, F4; NCT02065700, NCT03025308) receiving FIL $100 \mathrm{mg}$ (FIL100) QD, FIL $200 \mathrm{mg}$ QD (FIL200), adalimumab (ADA), methotrexate (MTX), or placebo (PBO) were included. Events related to statin use were analysed as exposed by treatment received. $\mathrm{N}$ and \% were provided.

Week (W)12 PBO-controlled safety analysis included pts receiving FIL100, FIL200, or PBO for $\leq 12 \mathrm{~W}$ (D1-2, F1-2); as-treated safety analysis included pts receiving long-term FIL100 QD ( $n=1647)$, FIL200 QD ( $n=2267)$, ADA ( $n=325)$, MTX $(n=416)$, or PBO $(n=781)(D 1-3, F 1-4)$; P3 as-randomised analysis included data up to W52 (F1-3) per assigned treatment.

Results: In each arm, similar proportions of pts took statins at baseline $(9.4 \%-11.9 \%)$; initiation during study was low $(1.2 \%-6.8 \%)$. Through W12 in PBO-controlled analysis, mean creatine phosphokinase (CPK; Figure 1), alanine aminotransferase (ALT), and aspartate aminotransferase (AST) levels were similar regardless of statin use and remained within normal levels across all arms.

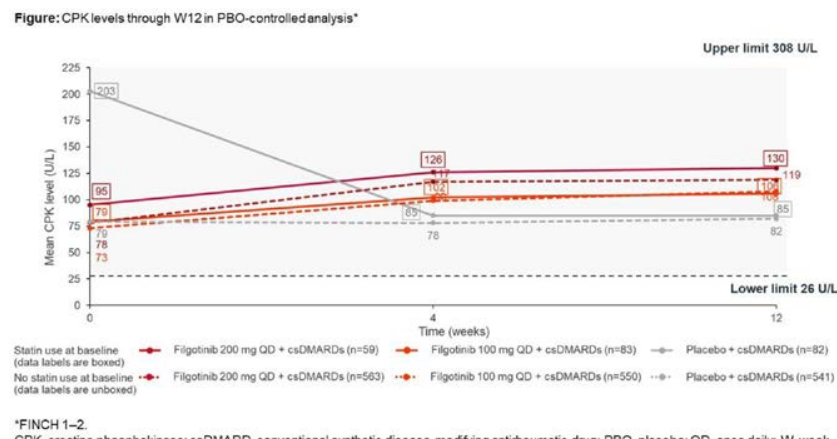

Mean baseline ALT and AST levels were 20-23 and 20-22U/L, respectively; at W12, ALT and AST ranged from 22-24 and 20-25U/L, respectively. Graded CPK, ALT, and AST elevations are in Table 1.

Table 1. Graded laboratory abnormalities at week 12 by baseline statin use in PBO-controlled analysis

\begin{tabular}{|c|c|c|c|c|c|c|}
\hline & \multicolumn{3}{|c|}{ Concomitant } & \multicolumn{3}{|c|}{ None } \\
\hline & $\begin{array}{l}\text { FIL200 } \\
(n=68)\end{array}$ & $\begin{array}{l}\text { FIL100 } \\
(n=95)\end{array}$ & $\begin{array}{c}\text { PBO } \\
(n=93)\end{array}$ & FIL200 (n=709) & $\begin{array}{l}\text { FIL100 } \\
(n=693)\end{array}$ & $\begin{array}{c}\text { PBO } \\
(n=688)\end{array}$ \\
\hline CPK increased* & 59 & 82 & 81 & 562 & 549 & 537 \\
\hline $\mathrm{G} 1(\leq 2.5 \times \mathrm{ULN})$ & $10(16.9)$ & $13(15.9)$ & $6(7.4)$ & $71(12.6)$ & $47(8.6)$ & $18(3.4)$ \\
\hline G2 (>2.5 to $5 \times$ ULN) & $3(5.1)$ & 0 & 0 & $6(1.1)$ & $2(0.4)$ & $3(0.6)$ \\
\hline G3 (>5 to $10 \times$ ULN) & 0 & 0 & 0 & $1(0.2)$ & 0 & $3(0.6)$ \\
\hline G4 $(>10 \times$ ULN $)$ & 0 & 0 & 0 & $1(0.2)$ & $2(0.4)$ & 0 \\
\hline AST increased ${ }^{\star *}$ & 68 & 94 & 92 & 708 & 692 & 684 \\
\hline G1 ( $\leq 3.0 \times U L N)$ & $9(13.2)$ & $11(11.7)$ & $7(7.6)$ & $97(13.7)$ & 79 (11.4) & $60(8.8)$ \\
\hline $\mathrm{G} 2(>3.0$ to $5.0 \times \mathrm{ULN})$ & 0 & 0 & 0 & $3(0.4)$ & $2(0.3)$ & $3(0.4)$ \\
\hline G3 (>5.0 to $20.0 \times$ ULN) & 0 & $1(1.1)$ & 0 & $2(0.3)$ & 0 & 0 \\
\hline G4 (>20.0xULN) & 0 & 0 & 0 & 0 & 0 & 0 \\
\hline ALT increased ${ }^{\star \star}$ & 68 & 94 & 92 & 708 & 692 & 684 \\
\hline G1 ( $\leq 3.0 \times U L N)$ & $13(19.1)$ & $14(14.9)$ & $13(14.1)$ & $98(13.8)$ & $92(13.3)$ & $72(10.5)$ \\
\hline G2 (>3.0 to $5.0 \times U L N)$ & 0 & $2(2.1)$ & 0 & $10(1.4)$ & $5(0.7)$ & $6(0.9)$ \\
\hline G3 (>5.0 to $20.0 \times$ ULN) & 0 & 0 & 0 & $1(0.1)$ & 0 & $1(0.1)$ \\
\hline G4 (>20.0xULN) & 0 & 0 & 0 & 0 & 0 & 0 \\
\hline
\end{tabular}

Data are $\mathrm{n}(\%)$. Grading per Common Terminology Criteria for Adverse Events v4.03

${ }^{*}$ FINCH 1-2**DARWIN 1-2, FINCH 1-2ALT, alanine aminotransferase; AST, aspartate aminotransferase; CPK, creatine phosphokinase; csDMARD, conventional synthetic disease-modifying antirheumatic drug; FIL200/100, filgotinib 200/100 mg + csDMARDs; Grade, G; PBO, placebo; ULN, upper limit of normal.

In the long-term as-treated analysis, $1(0.5 \%) / 6(3.2 \%) / 0 / 0 / 0$ treatment-emergent adverse events $(\mathrm{AE})$ of myalgia occurred in pts on statins at baseline receiving FIL200/FIL100/ADA/MTX/PBO and in $12(0.6 \%) / 8(0.5 \%) / 3(1.0 \%) / 2(0.5 \%) / 1$ $(0.1 \%)$ pts not on statins. Muscle spasms occurred in $2(0.9 \%) / 3(1.6 \%) / 1$ (3.2\%)/0/1 (1.1\%) pts on statins at baseline receiving FIL200/FIL100/ADA/MTX/ PBO and $21(1.0 \%) / 8(0.5 \%) / 0 / 3(0.8 \%) / 1(0.1 \%)$ pts not on statins at baseline. One patient not on statins receiving FIL200 reported rhabdomyolysis. For all treatment arms in P3 as-randomised analysis, mean LDL and HDL increased similarly from baseline (108-110 and $56-59 \mathrm{mg} / \mathrm{dL}$, respectively) to W52 (119130 and $59-71 \mathrm{mg} / \mathrm{dL}$, respectively).

Conclusion: No increases in statin-induced AEs such as muscle or liver toxicities occurred with statins and filgotinib coadministration; results are supported by a drug-drug interaction study. ${ }^{4}$ Mean LDL and HDL increased at W52 in all treatment arms.

REFERENCES:

[1] Genovese et al. JAMA. 2019;322:315-25.

[2] Westhovens et al. Ann Rheum Dis. 2021; online first.

[3] Combe et al. Ann Rheum Dis. 2021; online first.

[4] Anderson et al. EULAR 2021 abstract.

Disclosure of Interests: Peter C. Taylor Consultant of: AbbVie, Biogen, Eli Lilly, Fresenius, Galapagos, Gilead, GlaxoSmithKline, Janssen, Nordic Pharma, Pfizer, Roche, BMS, Sanofi, Celltrion, and UCB, Grant/research support from: Celgene, Eli Lilly, Galapagos, and Gilead, Christina Charles-Schoeman 
Consultant of: Gilead, Pfizer, and Regeneron-Sanofi, Grant/research support from: AbbVie, Bristol-Myers Squibb and Pfizer Inc, Muhsen Alani Shareholder of: Gilead Sciences, Inc., Employee of: Gilead Sciences, Inc., Mona Trivedi Shareholder of: Gilead Sciences, Inc., Employee of: Gilead Sciences, Inc., Vanessa Castellano Shareholder of: Gilead Sciences, Inc., Employee of: Gilead Sciences, Inc., Iyabode Tiamiyu Shareholder of: Gilead Sciences, Inc., Employee of: Gilead Sciences, Inc., Deyuan Jiang Shareholder of: Gilead Sciences, Inc., Employee of: Gilead Sciences, Inc., Lei Ye Shareholder of: Gilead Sciences, Inc., Employee of: Gilead Sciences, Inc., Sander Strengholt Shareholder of: Galapagos BV, Employee of: Galapagos BV, Michael Nurmohamed Speakers bureau: AbbVie, Bristol-Myers Squibb, Eli Lilly, Roche, and Sanofi, Consultant of: AbbVie, Celgene, Celltrion, Eli Lilly, Janssen, and Sanofi, Grant/research support from: AbbVie, Bristol-Myers Squibb, Celgene, Eli Lilly, Janssen, MSD, Mundipharma, Novartis, Pfizer, Roche, and Sanofi, Gerd Rüdiger Burmester Speakers bureau: AbbVie, Eli Lilly, Pfizer, and Gilead Sciences, Inc., Consultant of: AbbVie, Eli Lilly, Pfizer, and Gilead Sciences, Inc.

DOI: 10.1136/annrheumdis-2021-eular.933

\begin{tabular}{|l|l|}
\hline POS0661 & RAPID RESPONSE TO BARICITINIB IN PATIENTS \\
WITH RHEUMATOID ARTHRITIS AND AN INADEQUATE \\
RESPONSE TO METHOTREXATE AND AT LEAST \\
ONE BIOLOGIC DMARD: A CLINICAL AND POWER \\
DOPPLER ULTRASOUND STUDY
\end{tabular}

C. Baldi ${ }^{1}$, P. Falsetti ${ }^{1}$, E. Conticini ${ }^{1}$, S. G. Khayyat ${ }^{1}$, M. Bardelli ${ }^{1}$, S. Gentileschi ${ }^{1}$, L. Cantarini ${ }^{1}$, B. Frediani ${ }^{1}{ }^{1}$ Rheumatology Unit, University of Siena, Siena, Italy

Background: Baricitinib, an oral Janus kinase (JAK) 1-2 inhibitor, is currently used among biologic DMARDs (bDMARDs) after the failure of methotrexate (MTX) in rheumatoid arthritis (RA). Power Doppler ultrasound (PDUS) is a promising, non-invasive imaging method to assess synovitis in RA: results from numerous studies suggest that it provides additional information to clinical and conventional radiographic examinations.

Objectives: The main objective of our study was to evaluate short-term efficacy of Baricitinib in reducing synovitis, using the composite semi quantitative scale (0-3 grades) PDUS synovitis score, developed by the Outcome Measures in Rheumatology- European League Against Rheumatism (OMERACTEULAR)-Ultrasound Task Force. Moreover both synovial hyperplasia and intrasynovial power Doppler (PD) signal were also scored as single components/ parameters on 0-3 scales. Secondary objective was to assess the concordance between patient reported outcomes (PROs), markers of inflammation, physical examination and US.

Methods: We enrolled 30 patients fulfilling 2010 ACR and EULAR criteria for RA. All patients had failed at least one anti-TNF. Each patient was prescribed Baricitinib $4 \mathrm{mg} /$ daily at T0, in addition to MTX and/or oral steroids at a dosage $\leq 7,5 \mathrm{mg} /$ day of Prednisone or equivalent, at T'. All patients were evaluated at baseline (T0) and then after one month (T1), 3 months (T2) and 6 months (T3) of treatment. Swollen and tender joints (out of 28)were evaluated and recorded, as well as patient (PGA) and physician global assessment (PhGA) and pain, expressed in a visual analog scale (VAS). Disease activity was evaluated at each visit using DAS28 (Disease activity score 28), CDAI (Clinical disease activity index)and SDAI(Simplified disease activity index), accompanied by a complete blood count, Erythrocyte sedimentation rate (ESR) and C- reactive protein (CRP) collection. Statistical analysis was performed using GraphPad version 9.0.0. PDUS examination, was carried out by two rheumatologists (PF and $\mathrm{CB}$ ) blinded to clinical conditions of the patients, using an Esaote Mylab Twice (Genoa, Italy), equipped with a high-frequency (6-18 MHz) linear probe. With standardised Doppler parameters (pulse repetition frequency between 500-750 Hz; Doppler frequency between 7-11.1 MHz). PDUS was performed at each visit bilaterally for 22 joint sites [MCPs $1-5$, proximal interphalangeal joints (PIPs) 1-5, wrist, elbow, glenohumeral, knee, tibiotalar, talonavicular and calcaneocuboidal and metatarsophalangeal joints (MTPs) 1-5] for a total of 44 joints for each patient.

Results: we observed a reduction of VAS pain (T0 vs, T6<0,0001) PDUS composite score (T0 vs. T6 $p<0,0001$ ), Power Doppler (T0 vs. T6 $p<0,0001$ ) synovial hyperplasia (T0 vs. T6 $p=0,0002$ ), CRP (T0 vs. T6 $p<0,0001$ ) and ESR (T0 vs. T6 $p<0,0001$ ) was observed in our patients. Accordingly, DAS-28, CDAI and SDAI displayed a significant reduction too (DAS-28: T0 vs. T6 $p<0,0001$; CDAl: T0 vs. T6 $p<<0,0001$; SDAl: T0 vs. T6 $p=0,0003$ ).

Conclusion: We investigated the efficacy of Baricitinib in real life, evaluating both from a clinimetric and ultrasound point of view. Baricitinib, demonstrated a significant parallel and fast improvement in VAS, PDUS and CRP was found at follow up assessment as early as one month of therapy. In conclusion, these results demonstrated the short term efficacy of Baricitinib $4 \mathrm{mg}$ for up to 6 months and providing a prompt improvement of PROs within the first weeks of treatment.
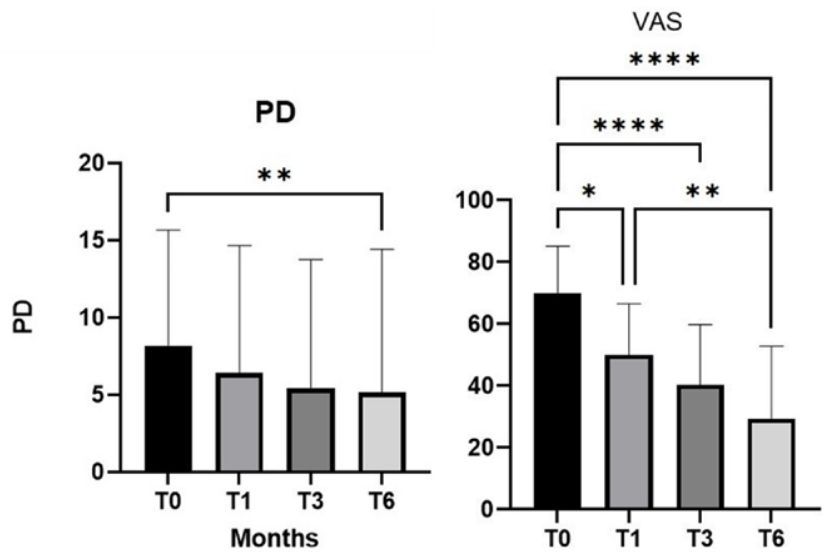

Figure 1. The difference between the means of PD and of the VAS pain over time (T0, T1 T3 and T6).Power Doppler (T0 vs. T6 ${ }^{\star *} p<0,0001$ ), VAS: (T0 vs. T1 ${ }^{*} p<0,0098 ; T 0$ vs. T3 ${ }^{* * * *}$ $\mathrm{p}<0,0001 ;$ T0 vs. T6 $\left.{ }^{\star \star \star *} \mathrm{p}<0,0001\right)$

Disclosure of Interests: None declared

DOI: 10.1136/annrheumdis-2021-eular.1007

\begin{tabular}{|l|l}
\hline POS0662 & PREDICTORS OF TOXICITY WITH \\
HYDROXYCHLOROQUINE AND CHLOROQUINE USE: A \\
SYSTEMATIC REVIEW
\end{tabular}

N. Citeroni ${ }^{1}$, S. Rampes ${ }^{1}$, P. Kotecha ${ }^{1}, J_{\text {J. Schoones }}^{2}$, E. Nikiphorou ${ }^{3,4}$, J. Galloway ${ }^{3,4}$. ${ }^{1}$ King's College London, GKT School of Medical Education, Faculty of Life Sciences \& Medicine, London, United Kingdom; ${ }^{2}$ Leiden University Medical Center, Walaeus Library, Leiden and the Hague, Netherlands; ${ }^{3}$ King's College London, Centre for Rheumatic Diseases, London, United Kingdom; ${ }^{4}$ King's College Hospital, Rheumatology Department, London, United Kingdom

Background: Hydroxychloroquine (HCQ) has attracted much attention especially during the COVID-19 pandemic. HCQ and closely related chloroquine (CQ) have known ocular and cardiac toxicity. However, although screening guidance now exists from the Royal College of Ophthalmologists for the former (RCOphth) ${ }^{1}$, little is known regarding predictors of both forms of toxicity.

Objectives: To systematically explore the literature on predictors of toxicity limited to cardiac and ocular toxicity.

Methods: A detailed search of the following databases was conducted: PubMed, Medline, Embase, Web of Science, The Cochrane library, EMCare and Academic Search Premier. Studies addressing predictors of $\mathrm{HCQ}$ toxicity with relevan search terms used were included. Exclusion criteria were: non-English articles, pre-clinical and paediatric studies. Three authors (SR, NC, PK) independently screened titles and abstracts for inclusion, ensuring each article was screened twice. Disagreement over inclusion was adjudicated by senior reviewers (EN, JG). Data extraction (SR, NC, PK) focused on predictors of toxicity.

Results: The search strategy retrieved 3103 studies. 147 studies were included for data extraction, of which 92 were eventually excluded due to: not identifying predictors $(n=17)$, reviews $(n=19)$, not ocular or cardiovascular toxicity $(n=18)$, case reports $(n=3)$, paediatrics $(n=1)$, screening articles that focused on detecting retinopathy $(n=30)$, article unobtainable at time of abstract submission $(n=4)$, leaving 55 studies for full review.

Studies addressing cardiac toxicity $(n=16)$ included: cohort, retrospective observational, a comparative pharmacovigilance, systematic monitoring protocol and a randomised control trial (RCT). The majority of these involved high-dose ( $>5 \mathrm{mg} /$ $\mathrm{kg} /$ day) use for acute COVID-19 infection. The main significant predictors identified were: Hydroxychloroquine (HCQ) use in combination with azithromycin (6/7 studies), cumulative dose (2 studies), pre-existing cardiovascular morbidity (2/3 studies) and prolonged baseline QTc (2 studies). Individual associations were also identified in the following: longer treatment duration, daily dosage, increased age, male gender, severe COVID-19 infection, abnormal liver function tests (LFTs) and concurrent use of loop-diuretics.

Regarding predictors of ocular toxicity $(n=39)$, only one study was a RCT. The remainder were observational studies: case-control, cohort, retrospective chart reviews and letters to the editor which included original patient data.

Several predictors of retinopathy and maculopathy were examined in two or more studies and included: duration of use (16/18 studies), daily dosage ( $7 / 13$ studies), cumulative dose (11/14 studies), increased age (10/11 studies), body weight or BMI (3/5 studies), renal impairment (5 studies), HCQ blood levels (2 studies), 